\title{
Mobile extracorporeal membrane oxygenation: Lead, follow, or get out of the way
}

\author{
Ashish S. Shah, MD
}

\author{
From the Department of Cardiac Surgery, Vanderbilt University Medical Center, Nashville, Tenn. \\ Received for publication Aug 21, 2016; revisions received Oct 20, 2016; accepted for publication Oct 28, 2016 \\ available ahead of print March 7, 2017 \\ Address for reprints: Ashish S. Shah, MD, Department of Cardiac Surgery, Vanderbilt University Medical Center, \\ 5025 Medical Center E, 1215 21st Ave St S, Nashville, TN 37232 (E-mail: ashish.s.shah@vanderbilt.edu). \\ J Thorac Cardiovasc Surg 2017;153:1369-70 \\ $0022-5223 / \$ 36.00$ \\ Copyright (c) 2017 by The American Association for Thoracic Surgery \\ http://dx.doi.org/10.1016/j.jtcvs.2016.10.107
}

Extracorporeal membrane oxygenation (ECMO) has been significant part of cardiothoracic surgical culture. Cardiac surgeons have had a monopoly on this technology for the better part of the last 50 years. This is no longer the case. The critical care community has embraced ECMO since the influenza pandemic in 2009, and this embrace has allowed the fundamental growth in this field to be lost to heart surgeons. Mobile ECMO is the latest example of an important innovation in public health that heart surgeons may have let slip away.

The first successful adult patient supported by ECMO was actually in a non-ECMO hospital and with a team of surgeons deployed "locally." That ECMO circuit was hardly portable, but the pioneering effort by J. Donald Hill and colleagues demonstrated mobility and possibility. ${ }^{1}$ Fast forward to 2016 and the newest ECMO circuits that have oxygenator, pump, and controls in a self-contained device. This remarkable integration has set the stage for "ECMO creep" — widening the application to cardiac arrest as alternative to traditional cardiopulmonary resuscitation. There are currently several clinical trials in Europe, Australia, and Japan that are examining ECMO cardiopulmonary resuscitation outcomes. ECMO creep also means moving out of the operating room and the cardiac intensive care unit to medical intensive care units, smaller hospitals, and finally airports, train stations, and art museums.

Mechanical support teams are increasingly organizing and structuring "ECMO transport" services. Here a hub ECMO center will go out to another hospital and either bring back a patient for potential ECMO or even cannulate at the distant facility. In the United States, the University of Michigan and Columbia groups have reported long-distance transport of patients supported by ECMO. ${ }^{2,3}$ All this has firmly established the reality of extending advanced support beyond hub hospitals. This is an urgent reality for today.

Importantly, there is an extension of ECMO to cardiac arrest in the prehospital setting, at the scene of the event. This represents truly local deployment of a team that cannulates victims in the field. Led by the group in Paris of Lamhaut and colleagues, ${ }^{4}$ a culture of "reanimation" has emerged.

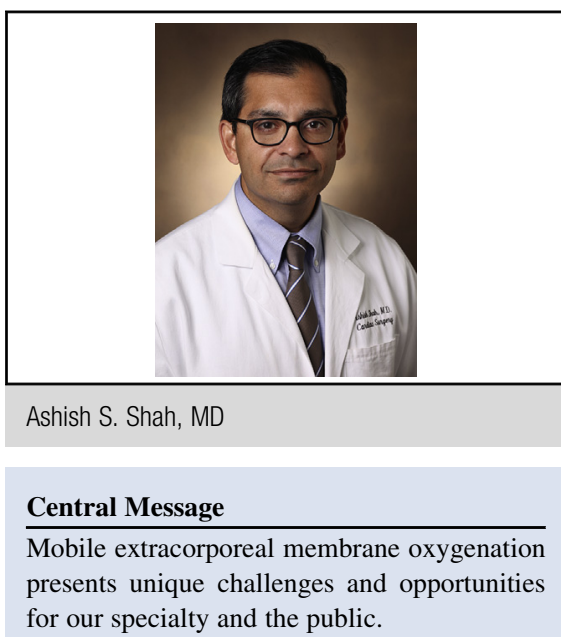

See Editorial Commentary page 1371.

Such emergency medical services have devised systems to initiate the call, dispatch a team in ECMO-equipped vehicles, and cannulate in public places.

As cardiac surgeons, we may dismiss the idea of cannulating with bad lighting and limited suction, but we do so at our own peril. The reanimators are not so intimidated, and they are making important contributions to our understanding of resuscitation. Moreover, the idea of truly local and public ECMO deployment expands this technology from a boutique, heroic anecdote to a public health issue.

Take the case of automated external defibrillators. In 2014, the California Ninth Circuit Court of Appeals examined the obligation of Target stores to provide automated external defibrillators. The premise of the complaint was that an automated external defibrillator is part of any public space, including large department stores. In this case, Verdugo $v$ Target Corporation, the court found in favor of the defendant. However, the opinion includes the following statement: "Judge Pregerson wrote separately to express his hope that Target will recognize its moral obligation to make Automatic External Defibrillators available for use in a medical emergency, and that the California legislature will take a hard look at the issue." 5 This statement is remarkable in 2 key ways. First, it suggests that complex but lifesaving medical devices have a place in public spaces. Second, with out-ofhospital sudden death survival abysmal $(<8 \%)$, what is the obligation to the public to offer something better?

And where are cardiothoracic surgeons in this area? This concept of resuscitative medicine, an arena that uses the 
tools-pumps, blood, temperature, whole-organ resuscitation - that we use every day, is noticeably devoid of surgeon input. In fact, the act of cannulation has been embraced by emergency medicine physicians and interventional cardiologists. Certainly, there is no way for cardiothoracic surgeons to be part of every mobile ECMO team, but it's remarkable that very little cardiac surgical input has been part of the conversation.

The recent report from the Düsseldorf group, ${ }^{6}$ as well as the pioneering work by Lamhaut and colleagues ${ }^{4}$ in Paris, has set the stage for the future. As described by Aubin and colleagues ${ }^{6}$ in JACC: Heart Failure, 115 patients were transported from outside facilities after a team locally deployed venoarterial ECMO in patients with cardiogenic shock or ongoing cardiopulmonary resuscitation. The patients were then transported to a central center. Aubin and colleagues ${ }^{6}$ of Düsseldorf report an impressive group of 381 -year survivors. Importantly, it was the cardiac surgery team that led this effort. Meanwhile, in Paris, Lamhaut is leading a clinical trial randomly allocating patients to prehospital ECMO versus transfer then ECMO for out-of-hospital arrest (NCT02527031). As our understanding of resuscitation from death progresses, we will need to answer 5 fundamental questions.

First, how do communities organize these programs? Should tertiary care medical centers form the hub, or should there be specialized ECMO or rescue units to handle the patients? The model will depend on the local needs, and input from the public will be important.

Second, who should receive ECMO resuscitation? Inclusion and exclusion criteria may seem straightforward, but on the fringes of sudden death we must consider "unknowables." Such unknowables as suicide, homelessness, and drug addiction all complicate out-of-hospital sudden death. Although the public is comfortable with the patient with an acute myocardial infarction who arrests, the patient with a hypothermic heroin overdose may be less socially acceptable—but no less salvageable.

Third, how should we resuscitate the dead? Despite a long history of perfusion science, cardiac surgeons still have ongoing disagreements on optimal temperature, oxygen, and flow at various stages of "reanimation" in everyday practice.

Fourth, how should communities (local, state, national, international) finance this enterprise? Estimated ECMO runs can potentially cost US $\$ 250,000$ to $\$ 500,000$ per patient for both survivors and nonsurvivors. ${ }^{7}$ This would be among the most expensive medical intervention budgeted for the public in history.

Finally, we will be forced to define futility. ECMO uniquely collides biologic, social, and moral forces to stress our understanding of futility and the public's expectations. There is an evolving discussion on futility in out-ofhospital arrest that is relevant. Emergency medical systems have developed termination of resuscitation criteria. The obvious goal is to avoid transport and resuscitation of patients who cannot survive; however, experts in this field have also expanded the discussion to include long-term disability in survivors and the problem of accidents caused by ambulances. In a landmark study from Canada, $30 \%$ of survivors had significant disability, including coma, at follow-up. ${ }^{8} \mathrm{~A}$ provocative letter from Millin and Galvagno ${ }^{9}$ highlights the unanticipated problems of disrupting crime scenes, the perils of lights and sirens, and suggests that prolonged resuscitations represent public harm. These are not trivial problems, and they further emphasize that there will be public expectations that need to be understood and incorporated in the goals of care. Interestingly, the failure to obtain a spontaneous rhythm in the field is a cornerstone of termination of resuscitation protocols. ECMO would potentially alter and eliminate this guideline. As the Paris group discovered, ${ }^{4}$ delaying ECMO was the key predictor of outcomes. Thus, when understanding futility, we will need to develop new guidelines that are not encompassed by our existing framework. This may be our most difficult question to answer.

Far away from our hybrid operating rooms and in the relatively terrifying domain of public spaces, there is a future for ECMO in the street. Although this may not be in our comfort zone, it is clear that others have embraced this new domain. It is a true opportunity for the entire medical community, but more importantly for cardiothoracic surgeons. No medical specialty is better prepared both intellectually, logistically, and technically to put mobile ECMO into operation.

In short, this is an opportunity to lead.

\section{Conflict of Interest Statement}

Author has nothing to disclose with regard to commercial support.

\section{References}

1. Hill JD, O'Brien TG, Murray JJ, Dontigny L, Bramson ML, Osborn JJ, et al. Prolonged extracorporeal oxygenation for acute post-traumatic respiratory failure (shock-lung syndrome)—use of the Bramson membrane lung. N Engl J Med. 1972:286:629-34.

2. Biscotti M, Agerstrand C, Abrams D, Ginsburg M, Sonett J, Mongero L, et al. One hundred transports on extracorporeal support to an extracorporeal membrane oxygenation center. Ann Thorac Surg. 2015;100:34-9; discussion 39-40.

3. Bryner B, Cooley E, Copenhaver W, Brierley K, Teman N, Landis D, et al. Two decades' experience with interfacility transport on extracorporeal membrane oxygenation. Ann Thorac Surg. 2014;98:1363-70.

4. Lamhaut L, Jouffroy R, Soldan M, Phillipe P, Deluze T, Jaffry M, et al. Safety and feasibility of prehospital extra corporeal life support implementation by non-surgeons for out-of-hospital refractory cardiac arrest. Resuscitation. 2013;84:1525-9.

5. Verdugo v Target Corp, supra, 704 F3d 1053 (2014) (Pregerson J, dissenting opinion).

6. Aubin H, Petrov G, Dalyanoglu H, Saeed D, Akhyari P, Paprotny G, et al. A suprainstitutional network for remote extracorporeal life support: a retrospective cohort study. JACC Heart Fail. 2016;4:698-708.

7. Maxwell BG, Powers AJ, Sheik AI, Lee PU, Lobato RL, Wong JK. Resource use trends in extracorporeal membrane oxygenation in adults: an analysis of the Nationwide Inpatient Sample 1998-2009. J Thorac Cardiovasc Surg. 2014;148:416-21.e1.

8. Morrison LJ, Visentin LM, Kiss A, Theriault R, Eby D, Vermeulen M, et al; TOR Investigators. Validation of a rule for termination of resuscitation in out-ofhospital cardiac arrest. $N$ Engl J Med. 2006;355:478-87.

9. Millin MG, Galvagno SM. More than 15 minutes of resuscitation prior to termination of resuscitation results in undue harm to the public health. Am J Emerg Med. 2016;34:1689-90. 